\title{
EDUCAÇÃO ALIMENTAR E NUTRICIONAL PARA GRUPOS VULNERÁVEIS: UM RELATO DE EXPERIÊNCIA DAS VIVÊNCIAS DE EXTENSÃO UNIVERSITÁRIA
}

\author{
FOOD AND NUTRITION EDUCATION FOR \\ VULNERABLE GROUPS: AN EXPERIENCE \\ REPORT OF UNIVERSITY EXTENSION \\ EXPERIENCES
}

\author{
Rosana Henn ${ }^{1}$ \\ Elisabeth Barth Almeida ${ }^{2}$ \\ Mariana Reiser Guedes ${ }^{3}$ \\ Alexandra Christiane Cabral dos Santos ${ }^{4}$ \\ Ana Paula Corteze Ott ${ }^{5}$ \\ Ana Júlia Vargas ${ }^{6}$ \\ Bruna Thais Pacheco $^{7}$ \\ Camila Thaís Andrade ${ }^{8}$ \\ Franciele Aparecida de Souza da Cruz ${ }^{9}$ \\ Gabriela Maria Natividade ${ }^{10}$ \\ Gabriella Dallago ${ }^{11}$ \\ Letícia de Souza Pessoa ${ }^{12}$
}

RESUMO: A Educação Alimentar e Nutricional é um campo de conhecimento e de prática contínua e permanente, transdisciplinar, intersetorial e multiprofissional que visa promover a prática autônoma e voluntária de hábitos alimentares saudáveis. Este trabalho objetivou relatar as práticas de extensão realizadas com usuários de dois CRAS, um CCI e um CMU do município de Itajaí-SC, no decorrer do ano de 2017, considerando a percepção dos acadêmicos envolvidos e a participação dos usuários assistidos por um projeto de extensão. Todos os materiais utilizados nas oficinas foram previamente elaborados pelas extensionistas, sob orientação das professoras envolvidas no projeto. Foram abordados assuntos relacionados à alimentação saudável, consumo de açúcares,

1 Nutricionista. Mestre em Ciências dos Alimentos pela Universidade Federal de Santa Catarina - UFSC. Professora titular da Universidade do Vale do Itajaí UNIVALI.E-mail: rhenn@univali.br.

2 Nutricionista. Mestre em Turismo e Hotelaria pela Universidade do Vale do Itajaí UNIVALI. Professora titular da UNIVALI. E-mail: bethbarth@univali.br.

3 Bacharel em Gastronomia. Mestranda em Saúde e Gestão do Trabalho pela Universidade do Vale do Itajaí - UNIVALI. Professora titular da UNIVALI. E-mail: marianaguedes@univali.br.

Licença CC BY: Artigo distribuído sob os termos Creative Commons, permite uso e distribuição irrestrita em qualquer meio desde que o autor credite a fonte original.
4 Acadêmica de Nutrição da Universidade do Vale do Itajaí - UNIVALI.

5 Graduada em Nutrição pela Universidade do Vale do Itajaí - UNIVALI.

6 Acadêmica de Nutrição da Universidade do Vale do Itajaí - UNIVALI.

7 Acadêmica de Nutrição da Universidade do Vale do Itajaí - UNIVALI.

8 Graduada em Nutrição pela Universidade do Vale do Itajaí - UNIVALI.

9 Acadêmica de Nutrição da Universidade do Vale do Itajaí - UNIVALI.

10 Acadêmica de Nutrição da Universidade do Vale do Itajaí - UNIVALI.

11 Acadêmica de Nutrição da Universidade do Vale do Itajaí - UNIVALI.

12 Acadêmica de Nutrição da Universidade do Vale do Itajaí - UNIVALI. 
gorduras e alimentos industrializados, mitos e verdades sobre alimentação, sazonalidade das frutas e exposição solar, alimentação e hidratação no verão. As atividades realizadas permitiram, a todos os envolvidos, a troca de saberes. Ressalta-se a importância do envolvimento acadêmico em projetos de extensão, o que possibilita o contato direto com a comunidade, permitindo que os conteúdos abordados durante a graduação estejam vinculados com a prática.

PALAVRAS-CHAVE: Educação Alimentar e Nutricional; Educação em Saúde; Promoção da Saúde; Prevenção de Doenças; Relações Comunidade-Instituição.

ABSTRACT: Food and Nutrition Education is an ongoing and permanent field of knowledge, and a transdisciplinary, intersectoral and multiprofessional practice that aims to promote the autonomous and voluntary practice of healthy eating habits. The aim of this study was to report extension practices performed with users of two CRAS - a CCI and a CMU in the city of Itajaí-SC, om 2017, considering the perceptions of the students involved and the participation of users assisted by an extension project. All the materials used in the workshops were previously prepared by the extension agents, under the guidance of the teachers involved in the project. Subjects related to healthy eating, consumption of sugars, fats and processed foods, myths and truths about food, seasonality of fruits and exposure to the sun, and food and hydration in the summer, were discussed. The activities carried out allowed all those involved to exchange knowledge. It is important to emphasize the importance of academic involvement in extension projects, which promotes direct contact with the community and enables the content addressed during the graduate studies to be linked to practical experience.

KEYWORDS: Food and Nutrition Education; Health Education; Health Promotion; Disease Prevention; Community-Institutional Relations.

\section{INTRODUÇÃO}

A Educação Alimentar e Nutricional (EAN) é um campo de conhecimento e de prática contínua e permanente, transdisciplinar, intersetorial e multiprofissional que visa promover a prática autônoma e voluntária de hábitos alimentares saudáveis. Conquistou mudanças conceituais e práticas significativas e importantes em seu processo de construção, considerando que atualmente a mesma está inserida no âmbito das políticas públicas, no contexto da promoção da saúde e da Segurança Alimentar e Nutricional (BRASIL, 2012).

É importante ressaltar ainda que a crescente importância da EAN no contexto da promoção da saúde e da alimentação saudável é vista como uma estratégia fundamental para enfrentar os novos desafios nos campos da saúde, da alimentação e da nutrição (MANCUSO; VINCHA; SANTIAGO, 2016). Além disso, as ações de EAN são fortes aliadas das atividades de extensão universitária.

A extensão universitária promove atividades de caráter educativo, cultural, científico e tecnológico, sendo essas desenvolvidas por meio de projetos que contemplam a articulação com o ensino e a pesquisa, abordando questões relevantes à sociedade (PEREIRA et al., 2015). Por meio da extensão ocorrem transformações sociais, assim como há influência na vivência das pessoas, abrindo caminhos para transformar a sociedade e a própria universidade. Com isso, entende-se que a extensão traz melhoria não somente ao público-alvo, mas para ambas as partes (DIVINO et al., 2013).

De acordo com o exposto, este trabalho objetivou relatar as práticas de extensão realizadas com usuários de dois Centros de Referência em Assistência Social (CRAS), um Centro de Convivência do Idoso (CCI) e um Centro de Múltiplo Uso (CMU) do município de Itajaí-SC, no decorrer do ano de 2017, considerando a percepção dos acadêmicos e dos professores envolvidos e a participação dos usuários assistidos por um projeto de extensão. 


\section{METODOLOGIA}

O presente trabalho trata de um relato de experiência que, de acordo com Gil (2008), é uma metodologia que dá margem para o pesquisador relatar suas experiências e vivências, lincando-as com o saber científico.

As ações de EAN foram realizadas por extensionistas e acadêmicos em estágio supervisionado obrigatório em Nutrição em Saúde Coletiva, do Curso de Nutrição, da Universidade do Vale do Itajaí (UNIVALI), sob orientação e supervisão de três docentes vinculadas ao Projeto de Extensão "Educação Alimentar e Nutricional para Promoção da Cidadania, da Saúde e Prevenção de Doenças”. Ressalta-se que a inserção dos acadêmicos em estágio obrigatório no projeto contempla a Curricularização da Extensão, proposta pelo Plano Nacional de Educação-PNE (BRASIL, Lei 13.005,2014). As ações foram realizadas com usuários de dois CRAS, de um CCI e um CMU do município de Itajaí-SC, na sua maioria idosos, entre abril e novembro de 2017. Essas ações foram registradas por meio de diário de campo, sempre preenchido pelas extensionistas ao final de cada atividade, com o intuito de facilitar o processo de organização de ideias e descrição do presente relato de experiência. Constavam no diário itens como: data, tema, número de participantes, pontos positivos e negativos, sugestões e comentários dos participantes.

Para planejamento, estruturação e avaliação das ações, os acadêmicos e os professores envolvidos mantiveram encontros quinzenais, nas quartas-feiras; já as ações educativas foram realizadas também quinzenalmente, sempre na semana subsequente às reuniões, nas terças e quartas-feiras.

\section{RELATO DA ORGANIZAÇÃO E DA REALIZAÇÃO DAS OFICINAS}

Inicialmente, foi estabelecido contato com os coordenadores dos CRAS, do CMU e CCI, para verificar o interesse e a viabilidade da realização de oficinas de EAN com os usuários vinculados aos locais. Em comum acordo, foram estabelecidas datas para realização dos encontros e, posteriormente, discutidos e definidos temas a serem abordados. Estes foram selecionados de acordo com as necessidades observadas nos locais.

O Quadro 1 demonstra as atividades realizadas, temas abordados e número de participantes no decorrer do ano de 2017.

Quadro 01: Atividades realizadas pelo projeto de extensão "Educação Alimentar e Nutricional para Promoção da Cidadania, da Saúde e Prevenção de Doenças”, locais e número de participantes. Itajaí-SC, 2017.

\begin{tabular}{|l|c|c|c|c|}
\multirow{2}{*}{ Atividades Realizadas } & \multicolumn{4}{c|}{ Locais } \\
\cline { 2 - 5 } & CRAS I & CRAS II & CCI & CMU \\
\hline Dez passos para uma alimentação saudável & - & 28 & 131 & 34 \\
\hline Mitos e verdades da alimentação & - & 19 & - & - \\
\hline Intestino saudável I & - & 19 & - & 29 \\
\hline Intestino saudável II & 09 & - & - & 34 \\
\hline Açúcares e gorduras & - & 21 & 63 & - \\
\hline Alimentos que melhoram o astral & - & 11 & 116 & - \\
\hline Prato saudável & - & 09 & 17 & - \\
\hline Frutas da primavera & 12 & - & - & - \\
\hline
\end{tabular}




\begin{tabular}{|l|c|c|c|c|}
\hline O consumo do açúcar & 13 & - & - & - \\
\hline Resgate dos hábitos alimentares & 09 & - & - & - \\
\hline Pré e probióticos & 13 & - & - & - \\
\hline Frutas da época & 06 & - & - & - \\
\hline Alimentação e azia & 10 & - & - & - \\
\hline As frutas da estação & 12 & - & - & - \\
\hline Exposição solar, alimentação e hidratação no verão & 8 & - & - & - \\
\hline O consumo dos alimentos industrializados & 13 & - & - & - \\
\hline TOTAL & $\mathbf{1 0 5}$ & $\mathbf{1 0 7}$ & $\mathbf{3 2 7}$ & $\mathbf{6 3}$ \\
\hline
\end{tabular}

Legenda: CRAS I: Centro de Referência em Assistência Social Nossa Senhora das Graças; CRAS II: Centro de Referência em Assistência Social Promorar; CMU: Centro Múltiplo Uso Promorar; CCI: Centro de Convivência do Idoso.

\section{DESCRIÇÃO DAS OFICINAS, DOS RESULTADOS E DA DISCUSSÃO}

Nogueira (2016) refere-se ao envelhecimento como um processo natural, espontâneo, progressivo e irreversível, que torna os indivíduos mais vulneráveis, podendo facilitar a ocorrência de processos patológicos. As patologias com maior incidência nessa população são as Doenças Crônicas Não Transmissíveis (DCNT), e elas têm uma grande probabilidade de serem desenvolvidas por hábitos alimentares inadequados, que vão causar prejuízos a essa população.

Diante da importância de estimular os hábitos alimentares saudáveis nesta população, as atividades do presente projeto de extensão foram voltadas a este fim. Todos os materiais utilizados nas oficinas foram previamente elaborados pelas extensionistas e acadêmicos em estágio obrigatório, sob orientação das professoras envolvidas no projeto.

Nos primeiros encontros em cada local, o projeto era exposto com uma breve introdução sobre os objetivos e a apresentação dos docentes e dos extensionistas, seguido pela oficina "Dez passos para uma alimentação saudável”, na qual foi utilizado um banner como material de apoio para exposição dos passos, baseados nos Guias Alimentares para População Brasileira (BRASIL, 2014; BRASIL, 2006). Além da exposição, as extensionistas esclareceram cada passo, dando abertura para um debate com os usuários.

Nesta oficina houve a participação de um bom número de usuários, os quais demonstraram interesse e conhecimento pelo assunto abordado. Entretanto, apresentaram muitas dúvidas do porquê de alguns alimentos não serem considerados saudáveis (embutidos, margarina, industrializados, entre outros) e resistência para aderir a mudanças nos hábitos alimentares. Nos dias de hoje, manter uma alimentação equilibrada está cada vez mais difícil devido à dieta inadequada e à formulação base dos alimentos processados (LOPES et al., 2017).

O tema "Mitos e verdades sobre alimentação" foi apresentado de maneira expositiva, no qual uma estagiária do curso de Nutrição pronunciava uma frase e os participantes respondiam se era mito ou verdade, usando uma plaquinha vermelha ou verde, respectivamente. Notou-se pouca participação na oficina, talvez pelo fato de que a atividade foi a primeira realizada com o público do CRAS II, que ainda estava acanhado frente à atividade.

Ações que contribuam para a educação em saúde, empoderamento e para o aumento da participação social são necessárias para contribuir com a busca de recursos para a promoção de 
saúde, além de serem de extrema importância para o processo de envelhecimento saudável e para a qualidade de vida (ALMEIDA et al., 2014).

Por este motivo, os profissionais de saúde assumem papel essencial no desenvolvimento de atividades educativas capazes de propor a prevenção de doenças e promoção da saúde, especialmente aos idosos, fornecendo, dessa forma, meios que permitam vencer os desafios impostos pela idade (ARAÚJO et al., 2013).

No tema "Intestino saudável" foram apresentados aos participantes alimentos benéficos e prejudiciais ao intestino e à ação destes alimentos. Em seguida, as figuras dos alimentos ficaram expostas em um banner que continha a imagem de um intestino saudável e a de um intestino doente e, ao final, foram esclarecidas as dúvidas. Os participantes demostraram interesse, participaram, esclareceram dúvidas e fizeram relatos sobre os seus hábitos alimentares e situação de saúde. Houve também a socialização de sugestões para futuros temas a serem discutidos. Ao final da ação, foi possível perceber que o conteúdo foi compreendido, apesar das dificuldades individuais, como o analfabetismo.

Em um segundo momento, a fim de reforçar o tema "Intestino saudável", visto que a maioria dos participantes relatou sintomas de constipação, foi realizado um jogo de tabuleiro com formato de intestino, dividido em "casinhas", em que cada uma representava um alimento que podia ser benéfico ou não. Os participantes foram divididos em dois grupos e o representante de cada grupo jogava o dado e avançava as casinhas. Em cada casinha (alimento) na qual o dado parava, falava-se sobre o alimento em questão. Apesar de ser estimulada a competição, como recompensa todos os grupos foram convidados a degustar um bolo de aveia com canela.

A dinâmica "Pré e probióticos" foi desenvolvida por meio de uma breve explicação sobre o tema e apresentados alimentos e produtos-fonte, a fim de destacar as propriedades e a vantagens do consumo de prebióticos e probióticos na alimentação cotidiana. Em seguida foi realizada uma brincadeira em que cada participante pegava um alimento e dizia se era um pré ou probiótico. Para finalizar, houve a degustação de um iogurte com frutas e chia. Os idosos interagiram, questionando e demonstrando interesse sobre o tema e relatando suas principais queixas a respeito do intestino e dos alimentos que percebem que são prejudiciais ao seu funcionamento.

A existência de uma microbiota saudável resulta em correto desempenho das funções fisiológicas do indivíduo, propiciando melhor qualidade de vida (DENIPOTE; TRINDADE, 2010). Por este motivo, é indispensável adotar uma dieta equilibrada e hábitos saudáveis, com mudanças comportamentais simples, como o aumento do consumo de alimentos ricos em fibras, aumento da ingestão de líquidos e prática de atividades físicas (GARCIA et al., 2016).

Diante da importância de uma alimentação equilibrada, foram realizadas as dinâmicas “Açúcares e gorduras" e "O consumo do açúcar”, demonstrando as quantidades em produtos industrializados de maior consumo entre o público-alvo, com ênfase nas consequências deletérias do consumo para a saúde. Percebeu-se grande participação dos envolvidos e expressões de surpresa com as quantidades de açúcar e gordura apresentados. O açúcar, quando consumido, além de promover sensação de bem-estar, em razão da liberação de dopamina e serotonina, ainda tem função adoçante e conservante nos alimentos. As gorduras e os óleos, por sua vez, contribuem para textura, aroma e maciez nos alimentos, ocasionando também a sensação de saciedade. Com isso, a inclusão destes componentes na indústria alimentícia e, consequentemente, na dieta humana, é muito significativa, porém um consumo desequilibrado de açúcares e óleos causa ao corpo humano 
deficiências nutricionais associadas a patologias como obesidade, hipertensão e diabetes, bem como danos à memória e à aprendizagem (SILVA et al., 2017).

Na oficina "Açúcares e gorduras" foram expostas embalagens de alimentos industrializados comumente consumidos pelos usuários dos locais (macarrão instantâneo, salgadinhos, refrigerantes, amendoim japonês, chocolate, gelatina, entre outros) e suas respectivas quantidades de açúcares e gorduras, demonstradas em recipientes, a fim de impressioná-los. Foi dada a oportunidade para que os usuários expusessem as impressões causadas pelo tema.

O tema "O consumo do açúcar" foi abordado na forma de um jogo de perguntas e respostas em que os participantes deveriam responder levantando um cartão verde, caso a resposta fosse sim e vermelho, para não. Teve o objetivo de esclarecer dúvidas sobre o consumo do açúcar, abordando as consequências, a diferença entre os tipos de açúcar e o diabetes. Além disso, mostrou a quantidade de açúcar nos produtos industrializados de consumo frequente entre o público-alvo. Também foi apresentado um cartaz com embalagens de produtos industrializados, com a respectiva quantidade de açúcar embaixo do alimento.

A dinâmica "Alimentos que melhoram o astral” foi realizada por meio de um bingo. Foram montadas cartelas com alimentos relacionados ao assunto, intercaladas por números. O primeiro participante a completar uma das colunas ou linhas da cartela foi premiado com uma cesta com frutas. Ao final da oficina, foi realizada a desgutação de um bolo, entregue sua receita, além de uma cartilha sobre Alimentação Saudável para o Idoso. Houve grande entusiasmo e interesse por parte dos idosos, o que pode ser percebido pelo número de participantes e das dúvidas e dos comentários realizados durante a atividade. A depressão é a doença psiquiátrica mais comum entre os idosos, a qual afeta sua qualidade de vida e tem se tornado um problema de saúde pública devido à sua elevada frequência. A orientação e o acesso a informações quanto à alimentação adequada, aliada à EAN, são estratégias capazes de minimizar problemas de saúde e melhorar a qualidade de vida, por meio da mudança de hábitos e comportamentos alimentares (MONTESO et al., 2012; SILVA; BARATTO, 2015).

$\mathrm{Na}$ atividade "Prato saudável”, os participantes foram divididos em grupos e orientados a montar um prato que consideravam saudável para o almoço, utilizando diferentes imagens de alimentos (arroz integral, feijão, salada crua e em conserva, carne frita, chás, café, refrigenrante, entre outros). Em seguida, deu-se início a uma roda de conversa e foi realizada a montagem de um prato saudável, utilizando um banner que continha um prato dividido com a proporção ideal de cada grupo alimentar. Nesta ocasião os idosos puderam demonstrar quais são os hábitos alimentares que prevalecem no seu dia a dia e esclareceram dúvidas a respeito do modo de preparo dos alimentos, distribuição, quantidade e composição das refeições.

A qualidade de vida de idosos está relacionada à autoestima e ao bem-estar pessoal e abrange desde o estado de saúde ao estilo de vida, incluindo os cuidados com a alimentação e o equilíbrio nutricional. Dessa forma, torna-se fundamental ter uma alimentação rica e variada do ponto de vista nutricional, a fim de proporcionar a longevidade com melhor qualidade de vida (MALTA; PAPINI; CORRENTE, 2013).

O tema "Frutas da época" foi trabalhado para incentivar os usuários a consumir frutas e respeitar sua sazonalidade, visto que na época elas ficam mais baratas, nutritivas e saborosas. Foram mostradas as frutas da época e os seus benefícios. Após a conversa, os participantes foram divididos em dois grupos e cada grupo teve que identificar a fruta, e colar em um cartaz de acordo com os seus benefícios. Com o mesmo objetivo da dinâmica anterior, foi realizada a atividade sobre 
“As frutas da estação", que foi trabalhada por meio de bingo. Como premiação, foi disponibilizado para degustação um bolo integral de frutas e sua receita foi entregue para todos os participantes. Ainda, em um terceiro momento, foi realizada uma roda de conversa sobre as "Frutas da primavera", destacando suas características nutricionais e o impacto na saúde, e também realizado um bingo e oferecido um bolo para degustação. Nesta, os idosos participaram ativamente, comentaram sobre suas frutas de preferência e a respeito dos benefícios que lhes foram explicados. Ainda, mostraramse satisfeitos com o bolo integral que lhes foi oferecido.

Segundo Janini, Bessler e Vargas (2015), dentre os fatores de risco na qualidade de vida dos idosos assistidos por uma Unidade Básica de Saúde no Rio de Janeiro, destacaram-se, para ambos os sexos, a alimentação inadequada, sendo que $95,83 \%$ das idosas e $85,71 \%$ dos idosos apresentam hábitos alimentares inadequados.

Pensando nisso, foi realizada a dinâmica "Resgate dos hábitos alimentares", com o intuito de repensar a alimentação. Os idosos foram receptivos e interessados, na atividade que teve como intuito demonstrar as mudanças da alimentação dos tempos antigos para os tempos modernos. Em um primeiro momento, foi solicitado que os participantes montassem um prato com recortes de alimentos que remetiam à infância. Após montarem seus pratos, cada participante apresentou o seu e relatou os motivos das escolhas daqueles alimentos. Posteriormente, foi realizada uma breve roda de conversa sobre as mudanças da alimentação dos tempos antigos comparadas aos tempos modernos.

Sobre o assunto "Alimentação e azia”, foi elaborado um painel dividido ao meio sendo "alimentos que irritam o estômago" e "alimentos que fazem bem ao estômago". Os idosos citaram os alimentos de cada grupo e, após, foi distribuído um folheto educativo sobre os alimentos explorados durante a atividade. Os idosos demonstraram interesse, esclareceram dúvidas e relataram seus hábitos alimentares e situação de saúde, além das experiências que vivem. Esta atividade também possibilitou a troca de conhecimentos e experiências acerca dos alimentos e seus efeitos à saúde. As orientações adequadas podem ajudar os idosos que lidam com o sintoma de azia a terem mais qualidade de vida, visto que esse sintoma, se ocorrido com frequência, interfere nas atividades habituais, além do desconforto causado ao indivíduo (KAHRILAS; SMOUT, 2012).

O tema "Exposição solar, alimentação e hidratação no verão" foi abordado em forma de roda de conversa, onde foram explicados e discutidos os tópicos: importância da exposição solar; vitamina D; cuidados com a alimentação no verão; importância de se manter hidratado e águas aromatizadas. Os resultados encontrados foram satisfatórios, pois ocorreu a interação de todos os participantes da roda com questionamentos acerca do tema proposto, além de perguntas pertinentes a outras dúvidas sobre alimentação saudável.

"O consumo dos alimentos industrializados" foi trabalhado pela exposição de cartaz com a embalagem de produtos industrializados expostos para a identificação, com a respectiva "quantidade de sal" embaixo dos alimentos. Foram abordados os malefícios que o consumo de alimentos industrializados podem acarretar à saúde, bem como a quantidade de sal que se deve consumir ao dia. Ao final, foi entregue um sachê com sal de ervas para cada participante. Os idosos foram participativos e se mostraram surpresos com a quantidade de sal presente nos produtos industrializados.

Pode-se salientar que mesmo com a baixa escolaridade percebida entre os participantes, foi grande o interesse, a curiosidade e a vontade de adquirir novos conhecimentos, tornando as atividades realizadas um momento de lazer e de troca de experiências. Vista a necessidade de avaliar sua percepção, foi realizada ao final das oficinas do segundo semestre uma pesquisa de 
satisfação, avaliada por meio de uma escala hedônica de quatro pontos: "muito ruim", "ruim", "bom" e "muito bom", sendo que a participação nesta avaliação não era obrigatória. Os resultados estão descritos no Quadro 2.

Quadro 02: Pesquisa de satisfação das ações realizadas pelo projeto de extensão "Educação Alimentar e Nutricional para Promoção da Cidadania, da Saúde e Prevenção de Doenças”. Itajaí-SC, 2017.

\begin{tabular}{|l|c|c|c|c|}
\hline Ações & Muito ruim & Ruim & Bom & Muito Bom \\
\hline Dez passos para uma alimentação saudável & - & - & 11 & 46 \\
\hline Açúcares e gorduras & - & - & 05 & 31 \\
\hline Prato saudável & 01 & 01 & 08 & 40 \\
\hline Total & 01 & 01 & 24 & 117 \\
\hline
\end{tabular}

\section{CONSIDERAÇÕES FINAIS}

A promoção da EAN, por meio de ações voltadas para um público específico e aplicadas de forma dinâmica, é uma estratégia que leva em consideração particularidades de cada população, devendo ser de fácil entendimento, envolvendo os participantes no contex to e fazendo com que o aprendizado ocorra de maneira simples e espontânea.

As atividades realizadas permitiram a todos os envolvidos, desde professores, extensionistas, estagiários e público-alvo do projeto, a trocas de saberes, os desafios na aplicação das oficinas, o acesso a informações seguras sobre alimentação, que serviram como ferramentas para desmistificar conceitos estabelecidos previamente. Tal contexto permitiu aos participantes o esclarecimento de suas dúvidas e possibilitou aos acadêmicos o contato com a realidade da comunidade, conhecendo seus desafios e limitações, promovendo a adesão a mudanças alimentares e melhora da qualidade de vida.

Dentre as dificuldades encontradas destaca-se a resistência dos participantes em deixar de lado as atividades que estavam realizando nos locais de atuação do projeto de extensão, muitas vezes de lazer (dança, bordado, pintura, jogo de cartas) para participar das atividades de EAN. Esse fato tornou-se um desafio a ser vencido durante o planejamento e a elaboração das ações, que sempre visaram despertar o interesse dos participantes pelas dinâmicas. Essas se tornaram cada vez mais interativas, com maior participação dos envolvidos, facilitando o processo de aprendizagem e a troca de experiências.

Ressalta-se a importância do envolvimento acadêmico em projetos de extensão, o que possibilita o contato direto com a comunidade, permitindo que os conteúdos abordados durante a graduação estejam vinculados com a prática, e faz com que a vivência da extensão seja um espaço para a formação do futuro profissional, desenvolvendo o ato de cidadania por meio de responsabilidade social para grupos vulneráveis. 


\section{REFERÊNCIAS}

ALMEIDA, L. F. F. et al. Promoção da saúde, qualidade de vida e envelhecimento - A experiência do projeto "Em Comum-Idade: uma proposta de ações integradas para a promoção da saúde de idosos das comunidades de Viçosa-MG". Elo - Diálogos em Extensão, v.3, n.2, p.71-80, 2014.

ARAÚJO, V. S. et al. Nexus and challenges of health education for elderly in primary care. Journal for Nursing - UFPE Online, v.7, n.5, p.1311-1318, 2013.

BRASIL. Ministério do Desenvolvimento Social e Combate à Fome. Marco de referência de educação alimentar e nutricional para as políticas públicas. Brasília: MDS, 2012. Disponível em: <https:// www.ideiasnamesa.unb.br/files/marco_EAN_visualizacao.pdf>. Acesso em: 01 nov. 2017.

BRASIL. Ministério do Desenvolvimento Social e Combate à Fome. Lei Orgânica da Assistência Social. Brasília: MDS, 2009. Disponível em: <http://www.mds.gov.br/webarquivos/publicacao/assistencia_ social/Normativas/LoasAnotada>Acesso em: 01 nov. 2017.

BRASIL. Lei 13.005, de 25 de junho de 2014. Aprova o Plano Nacional de Educação - PNE e dá outras providências. Disponível em: < http://www.planalto.gov.br/ccivil _03/Ato2011- 2014/2014/ Lei/L13005.htm>. Acesso em: 05 dez. 2017.

BRASIL. Lei $n^{\circ} 4345$ de 24 de junho de 2005. Dispõe sobre benefícios eventuais aos cidadãos carentes, criação do cartão cidadania, e dá outras providências.

BRASIL. Ministério da Saúde. Guia Alimentar para a população brasileira: promovendo a alimentação saudável. Brasília: Ministério da Saúde, 2014.

DENiPOTE, F. G.; TRINDADE, E. B. S. de M.; BURINI, R. C. Probióticos e prebióticos na atenção primária ao câncer de cólon. Arquivos de Gastroenterologia, v. 47, n. 1, p. 93-98, 2010.

DIVINO, A. E. do A. A extensão universitária quebrando barreiras. Cadernos de Graduação, v. 1, n.16, p. 135-140, 2013.

GARCIA, L. B. et al. Constipação Intestinal: Aspectos Epidemiológicos e Clínicos. Saúde e Pesquisa, v. 9, n. 1, p. 153-162, 2016.

GIL, A. C. Como elaborar projetos de pesquisa. 5.ed. São Paulo: Atlas, 2008.

JANINI, J. P.; BESSLER, D.; VARGAS, A. B. de. Educação em saúde e promoção da saúde: impacto na qualidade de vida do idoso. Saúde em Debate, v. 39, n. 105, p. 480-490, 2015.

KAHRILAS, P. J.; SMOUT, A. J. P. M. Transtornos esofágicos. Arquivos de Gastroenterologia, v. 49, 2012.

LOPES, B. de C. et al. Atividades de conscientização sobre o consumo equilibrado de açúcares, sais, óleos e gorduras. II Encontro de Iniciação Acadêmica. Encontros Universitários da UFC, Fortaleza, v. 2 , n. 1, p.4937-4937, 2017.

MALTA, M. B.; PAPINI, S. J.; CORRENTE, J. E. Avaliação da alimentação de idosos de município paulista - aplicação do Índice de Alimentação Saudável. Ciência \& Saúde Coletiva, v. 18, n. 2, p. 377- 384, 2013.

MANCUSO, A. M. C.; VINCHA, K.R.R.; SANTIAGO, D. A. Educação Alimentar e Nutricional como prática de intervenção: reflexão e possibilidades de fortalecimento. Physis: Revista de Saúde Coletiva, Rio de Janeiro, v. 26, n. 1, p. 225-249, 2016. 
MONTESO P, F. C, et al. Depression in the elderly: study in a rural city in southern Catalonia. Journal of Psychosocial and Mental Health Nursing, v.19, n.5, p. 426-429, 2012.

NOGUEIRA, L. R. et al. Avaliação Qualitativa da Alimentação de Idosos e suas Percepções de Hábitos Alimentares Saudáveis. Journal of Health Sciences, v. 18, n. 3, p. 163-70, 2016.

PEREIRA L. M., et al. Relato de experiência: a pesquisa de campo no âmbito da extensão universitária. Revista Intercâmbio, v. 6, n.1, p.149-155, 2015.

SILVA, A. A. da et al. Ações educacionais em instituições públicas e privadas de conscientização sobre o consumo excessivo de açúcares e óleos. XXVI Encontro de Extensão. Encontros Universitários da UFC, v. 2, p. 3587-3587, 2017.

SILVA, J. V.; BARATTO, I. Nutrição: avaliação do conhecimento e sua influência em Universidade aberta a terceira idade. Revista Brasileira de Obesidade, v. 9, n. 53. p. 176-187, 2015. 